\title{
Effects of turbid media optical properties on object visibility in subsurface polarization imaging
}

\author{
Ralph E. Nothdurft and Gang Yao
}

\begin{abstract}
We studied the effectiveness of using polarized illumination and detection to enhance the visibility of targets buried in highly scattering media. The effects of background optical properties including scattering coefficient, absorption coefficient, and anisotropy on image visibility were examined. Both linearly and circularly polarized light were used in the imaging. Three different types of target were investigated: scattering, absorption, and reflection. The experimental results indicate that target visibility improvement achieved by a specific polarization method depends on both the background optical properties and the target type. By analyzing all polarization images, it is possible to reveal certain information about target or the scattering background. (C) 2006 Optical Society of America
\end{abstract}

OCIS codes: $260.5430,110.7050,290.1350$.

\section{Introduction}

Polarization is an intrinsic property of light. ${ }^{1}$ While human vision cannot sense polarization states, some other species may have the capability of utilizing polarization in their vision systems. ${ }^{2}$ To extend human vision capabilities, polarization video imaging is developed by incorporating polarization optics into a conventional video imaging system to selectively choose illumination polarization states and detect specific polarized backscattering light. ${ }^{3-7}$ It has been found that polarization imaging can enhance target visibility in a scattering environment. Many studies have applied polarization gating to reject surface glare when probing for deep targets, ${ }^{5,7}$ or to selectively detect photons from superficial layers of scattering media. ${ }^{8}$ It was demonstrated that polarization imaging can reveal hidden objects that cannot be seen with regular imaging. ${ }^{9,10}$ In addition, polarization techniques can be utilized to enhance degraded scenic images due to haze effects. ${ }^{11}$

Besides improving imaging contrast in turbid media, polarization imaging has also been applied to characterize material properties in computer vision. ${ }^{12-14}$ Chen and Wolff ${ }^{14}$ used the phase information of

The authors are with the Department of Biological Engineering, University of Missouri-Columbia, Columbia, Missouri 65211. G. Yao's e-mail address is yaog@missouri.edu.

Received 1 November 2005; revised 19 January 2006; accepted 2 February 2006; posted 9 February 2006 (Doc. ID 65729).

0003-6935/06/225532-10\$15.00/0

(C) 2006 Optical Society of America specularly reflected polarized light to discriminate between metal and dielectric materials in clear media. For applications in highly scattering media such as tissues, Demos et al. ${ }^{15}$ reported that bulk pathological tissues had a higher depolarization ratio than normal tissues. Jacques et al. ${ }^{16}$ further demonstrated that polarization-sensitive detection could discriminate different pathological features in skin. Although their experimental results are encouraging, the detailed mechanisms behind these phenomena require further investigation ${ }^{17}$ to fully explore the potential of this technique.

Linearly polarized light is usually used in polarization imaging because it is easy to calibrate and handle. However, it has long been realized that circularly polarized light propagates differently in turbid media than in linearly polarized light. ${ }^{18-20}$ For media with greater anisotropy, backscattered light tends to preserve the original polarization state when circularly polarized light is used, which is referred to as polarization memory. ${ }^{21-23}$ This effect is induced by a series of small angle forward-scattering events ${ }^{22}$ experienced by the backscattered light. Very recently, it has been demonstrated ${ }^{23,24}$ that circular cross-polarized light can improve image contrast due to polarization memory effects. It still remains a question as to whether a specific polarization component can be universally applied and to what extent polarization memory can be useful.

A thorough understanding of the contrast mechanisms involved in polarization imaging is essential for data interpretation in practical applications. This is especially important when identifying a specific 


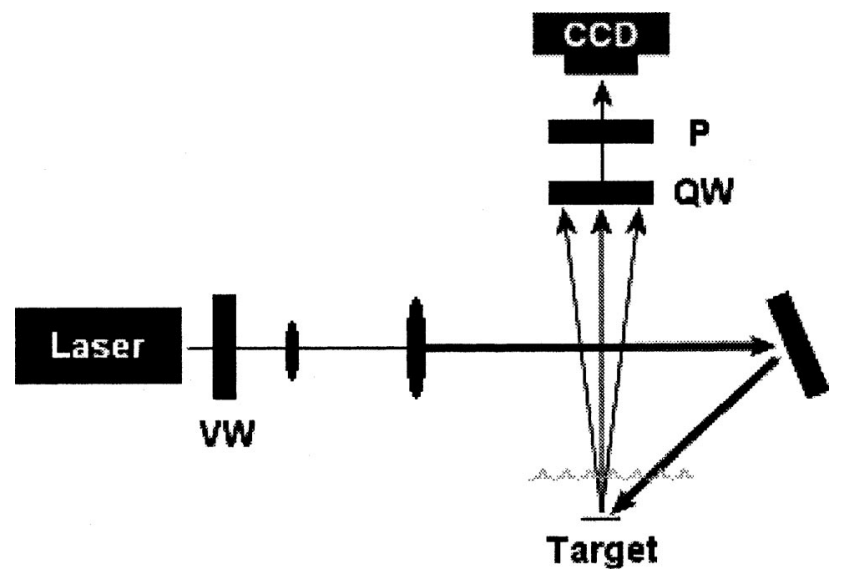

Fig. 1. Schematic of the experimental setup: $\mathrm{P}$, polarizer; $\mathrm{QW}$, quarter-wave plate; VW, variable wave plate.

type of material becomes the key objective in imaging. A recent simulation study ${ }^{25}$ showed that different types (scattering, absorption, and reflection) of object appeared differently in subsurface polarization imaging in turbid media, which implied that polarization detection could be used to identify different material types. This was later verified in experiments ${ }^{26}$ using Intralipid as the scattering phantom. However, because of the multidisperse nature of Intralipid, ${ }^{27}$ polarization memory effects did not present in the experiments. In addition, it is not clear how variation in background optical properties affects polarization image visibility of different optical targets.

We studied the effects of background optical properties on polarization imaging visibility. Three types of target were studied: reflecting, scattering, and absorption. These target represent major object types and contrast mechanisms in optical imaging. The effects of background absorption, scattering, and anisotropy on the visibility of various polarization images were investigated. We show that the effectiveness of polarization imaging, including the polarization memory effect, is sensitive to the background optical properties. For example, the circular cross-polarized light may provide superior image enhancement in one sample and become inferior in other samples. Most importantly, these changes are target-type dependent.

\section{Materials and Methods}

\section{A. Image Acquisition}

The experimental setup (Fig. 1) is similar to what has been used in a previous study. ${ }^{26}$ We used a $20 \mathrm{~mW}$ polarized He-Ne laser (633 nm wavelength) as the light source. A beam expander was used to expand the laser beam size so that it can illuminate a $20 \mathrm{~mm}$ sample area. The laser was incident upon the sample at a $45^{\circ}$ angle. A variable wave plate (VW) was used at the laser output to select either linearly or circularly polarized light. This wave plate can be fine tuned to compensate for polarization changes induced by other components in the system. Backscat- tered light from the phantom was imaged at normal incidence by a CCD camera (Pulnix TM-7AS). A quarter-wave plate $(\mathrm{QW})$ and a linear polarizer $(\mathrm{P})$ were mounted in front of the camera so that polarized images can be captured. The polarization precision was carefully calibrated using a mirror. We measured that our system can achieve polarization extinction ratios of $0.05 \%$ for both linearly and circularly polarized light.

The camera captures $640 \times 480$ 8-bit gray-scale images. The camera aperture accepted photons within $1.7^{\circ}$ over a $16 \mathrm{~mm} \times 12 \mathrm{~mm}$ imaging area, while the imaging depth was sufficient to cover the full range of heights measured. To reduce electronic noise, the camera is set to minimum gain and shutter speed is adjusted so that the brightest image can occupy the full 8-bit dynamic range. Camera settings are then held constant throughout the investigation. The captured data were recorded and processed by a computer using a custom software program written with LabVIEW (National Instruments, Austin, Texas), Speckle noise ${ }^{28}$ resulting from coherent illumination is dealt with by multiply averaging a sequence of $N$ captures. For our arrangement, an $N$ of 100 was sufficient to reduce speckle-induced variations to $\sim 1 \%$ of the mean pixel value. As a practical way to eliminate any inhomogeneous illumination effects, a base image was taken for each testing phantom without a target present. After smoothing with a Gaussian kernel, this base image was used to normalize the other images acquired from the same phantom. Such normalization was applied to the raw images prior to analysis.

\section{B. Scattering Phantom}

Scattering phantoms consisted of polystyrene microspheres (Polysciences Incorporated, Warrington, Pennsylvania) of different sizes in distilled water. Diluted Indian ink was added to introduce absorption. We calculated sample anisotropy $g$ and scattering coefficient $\mu_{s}$ using Mie theory. Microspheres of 0.989 and $0.356 \mu \mathrm{m}$ diameters were chosen to yield anisotropy $g$ values of 0.92 and 0.72 , respectively. Different concentrations of microspheres were used to obtain scattering coefficients from $\sim 10$ to $150 \mathrm{~cm}^{-1}$. The absorption coefficients used in this study were from 0.1 to $0.4 \mathrm{~cm}^{-1}$. These optical properties were chosen to be consistent with applications in biological imaging. However, results reported in this study should be applicable to the more general concept of polarization imaging.

Three types of $3 \mathrm{~mm} \times 3 \mathrm{~mm}$ target were used as embedded objects in the scattering phantom. They represented three distinct material types: reflecting, scattering, and absorption. The reflecting target was a microscope coverslip sputter coated with a $120 \mathrm{~nm}$ layer of platinum. The other targets were plastic backing coated with a thick layer of model paint. Black paint and white paint were used to simulate highly absorbing and highly scattering targets, respectively. The target was mounted on a thin glass 
sheet. Our testing indicated that the glass sheet did not measurably influence the experimental results. The depths of the target can be adjusted by a vertical stage at a step size of $0.2 \mathrm{~mm}$ with a $0.01 \mathrm{~mm}$ resolution. Because the medium depth below the target was significantly greater than the target depths examined, the phantoms can be treated as semi-infinite media. Zero depth was measured relative to the phantom surface and was the predominant source of error owing to variation among the phantoms and human judgment. Values reported in this paper are reliable to within a \pm 1 data point. In the results, the target depth is represented in transport mean-free-path (mfp) units, defined as $1 / \mu_{s}{ }^{\prime}$ or $1 / \mu_{s}(1-g)$.

\section{Polarization Imaging}

All images shown correspond to areas of $12 \mathrm{~mm} \times$ $12 \mathrm{~mm}$. A total of ten images are obtained for each phantom at a specific target depth. Four raw component images can be measured directly in the experiments by adjusting the wave plates and polarizers. They are linearly and circularly copolarized $(\mathrm{CO})$ and cross-polarized (CR) images postnormalization. The pixel values for the components were from 0 to 255 because of the 8-bit digitization. The summation of $\mathrm{CO}$ and $\mathrm{CR}$ components is the equivalent of an unpolarized (UNPOL) image, whose pixel values also represent illumination. The unpolarized images formed by linear and circular illumination were compared to ensure they are identical. There are two additional images that can be calculated from both linearly polarized light and circularly polarized light: differential polarization (DIFF) image, and degree of polarization (POL) image. The DIFF image was calculated as

$$
\mathrm{DIFF}=\mathrm{CO}-\mathrm{CR} .
$$

The corresponding pixel values were from -255 to 255. The POL image was calculated as

$$
\mathrm{POL}=\frac{\mathrm{DIFF}}{(\mathrm{CO}+\mathrm{CR})}
$$

The corresponding pixel values were from -1.0 to 1.0 . We did not use absolute values so that the relative weight of those two orthogonal components can be evaluated. The DIFF image is the more complicated of the two because it is affected by both polarization and illumination, whereas the POL is unaffected by the latter. The DIFF calculation preferentially selects the least depolarized photons, while the POL represents the degree of copolarization. When interpreting the images, it is important to realize that image appearance is determined by the relative pixel values between target and background. For example, a target in the POL image appears dark when the light from the target region had a lower copolarization degree than the surrounding media.

\section{Calculation of Image Visibility}

Image visibility was assessed using the contrast between target and background and the background noise level:

$$
\text { visibility }=\frac{|\bar{T}-\bar{B}|}{\sigma_{B}}
$$

where $\bar{T}$ is the mean intensity of the target; $\bar{B}$ is the mean intensity of the image background; and $\sigma_{B}$ is the standard deviation of the background image intensity. The target region $T$ was measured by using pixels from the middle quarter of the target, while the background region $B$ was assessed using imaging areas from the four corners of the image. The $\sigma_{B}$ value provides an estimate of the background image noise, which ultimately limits the target visibility even if the histogram of the image is rescaled. This is similar to the signal-to-noise limitation in signal processing. Pixel value variations in acquired images can be induced by many sources, for example, electronic noise, residual speckle noise, laser power and distribution fluctuation, and quantum noise in incident light. In our arrangement, $\sigma_{B}$ is found to be primarily correlated with pixel intensity. Therefore the visibility measurement is similar to the traditional image contrast for illumination-based raw component images. ${ }^{25,26}$ We found that this visibility assessment provides very good agreement for visual results.

\section{Results}

The target appearance in an unpolarized image depends on the background optical properties. In addition, the same target appears differently in different polarization images. Figure 2 shows an example where polarization imaging is applied to image three

\section{Linear polarization}

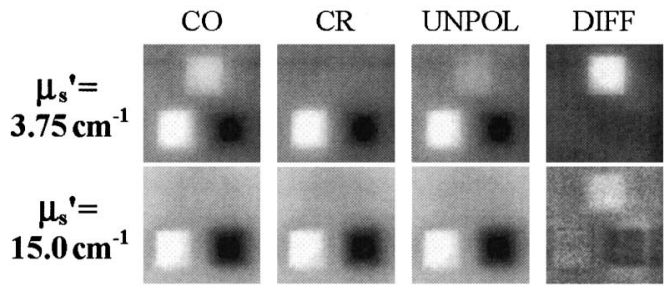

\section{Circular polarization}
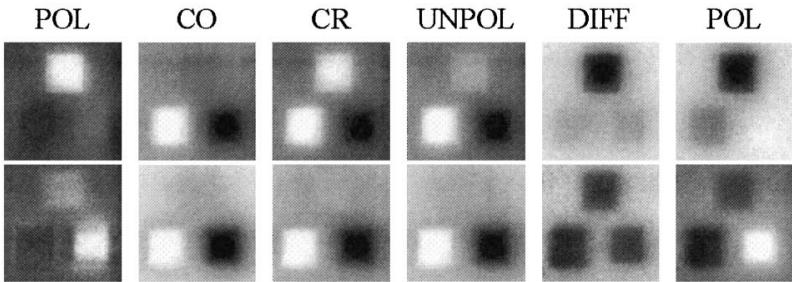

Fig. 2. Polarization images of combined multiple targets in the scattering media of two different scattering coefficients. The absorption coefficient is $0.1 \mathrm{~cm}^{-1}, g=0.72$. The reflection target is placed at the top of the image, and the scattering and absorption targets are located at the bottom left and right, respectively. Physical depth was adjusted to maintain an optical depth of $0.9 \mathrm{mfp}^{\prime}$. 
(a)

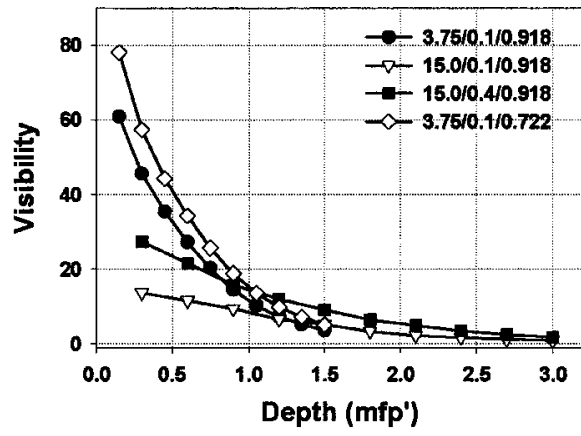

(b)

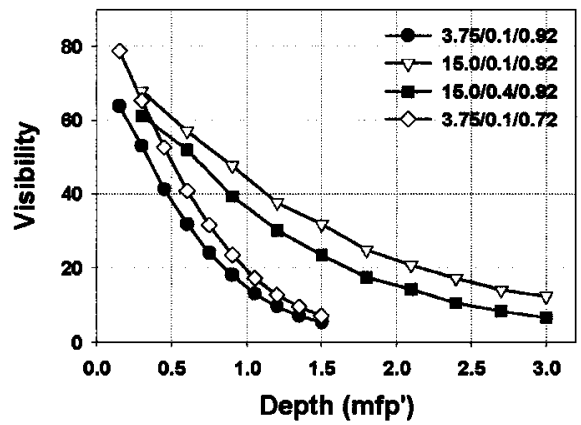

(c)

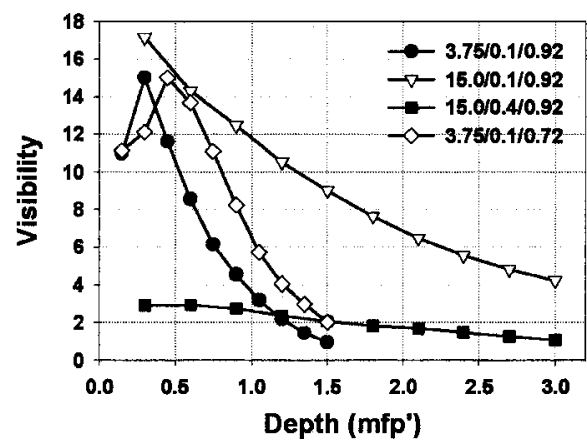

Fig. 3. Effects of background optical properties on an unpolarized image visibility of (a) scattering target, (b) absorption target, and (c) reflection target. The background optical properties are listed in the figure legend as $\mu_{s}{ }^{\prime} / \mu_{a} / g$.

different types of target: scattering, absorption, and reflection. The background turbid media is made of $0.356 \mu \mathrm{m}$ microspheres at different concentrations. The absorption coefficient is $0.1 \mathrm{~cm}^{-1}$. The three targets are located at $0.9 \mathrm{mfp}^{\prime}$. For image display, the local palette of each image was linearly rescaled to the full 8-bit range available without further processing.

A reflection target can specularly reflect the incident light. It preserves the polarization of linearly polarized light, and reverses the helicity of the circular polarized light. The reflection target has very low visibility in an unpolarized image at low-scattering coefficients and is invisible at high-scattering coefficients. However, it is significantly enhanced in DIFF and POL images with a dark appearance in the circular DIFF and POL, and a bright appearance in the linear DIFF and POL. A scattering target reflects incident light and randomizes the polarization; whereas an absorption target absorbs all the incident light. The absorption and scattering targets have low visibilities in linear DIFF and POL images, but are clearly visible in circular DIFF and POL images. The scattering target appears dark in both circular DIFF and POL images; the absorption target appears dark in circular DIFF and bright in circular POL images.

It can be seen that the appearance of a specific target in scattering media is relatively stable, especially in the DIFF and POL images. However, their image visibilities are significantly changed at different background optical properties. Therefore it is necessary to examine such effects in detail to determine the one or more polarization imaging components that can be applied to enhance the contrast of a specific target.

\section{A. Effects of Background Optical Properties on Unpolarized Images}

One key application of polarization imaging is to improve object visibility over unpolarized images. Therefore the unpolarized image provides an inherent mechanism for judging performance. The visibility of unpolarized images depends on the relative illuminance from the target and the background medium. The effects of background optical properties on diffuse reflectance from a homogeneous medium are well defined. In the diffuse regime of high scattering, the total diffuse reflectance from a turbid medium is a function of the $\mu_{s}{ }^{\prime} / \mu_{\alpha}{ }^{29}$ The increased $\mu_{s}{ }^{\prime}$ increases the background illumination. Increasing $\mu_{a}$ to restore the $\mu_{s}{ }^{\prime} / \mu_{a}$ ratio brings the background back down. The reduction in $g$ has a negligible effect on unpolarized visibility when comparing phantoms with the same $\mu_{s}{ }^{\prime}$. The signal from the target is more complicated than the signal from the background. It has contributions from both photons that have interacted with the target and background photons that have not interacted with the target. Near the surface, the target signal is mainly influenced by the target properties. As the target depth increases, the contributions from background photons become increasingly important and eventually overwhelm the photons carrying target information.

The background optical properties have different effects on different targets. Because the scattering target is always brighter than the background, an increase in background illumination reduces the visibility of the scattering target [Fig. 3(a)]. At low $\mu_{s}^{\prime}=3.75 \mathrm{~cm}^{-1}$, the background illumination is substantially lower than the backscattered light from the object, as a result the scattering target stands out quite dramatically. In the phantom of a high $\mu_{s}{ }^{\prime}=15 \mathrm{~cm}^{-1}$ and a low $\mu_{a}=0.1 \mathrm{~cm}^{-1}$, the background is almost as bright as the target, so the signal is quite weak by comparison. When we restore the $\mu_{s}{ }^{\prime} / \mu_{a}$ ratio in the high $\mu_{s}{ }^{\prime}$ and the high $\mu_{\alpha}$ phantom, both the target and the background are reduced; however, the effect is most significant for the background.

Conversely, the visibility of the absorbing target improves with increased background reflectance [Fig. 3(b)]. This is because the signal from the absorption object is always smaller than the background. Thus 
(a)

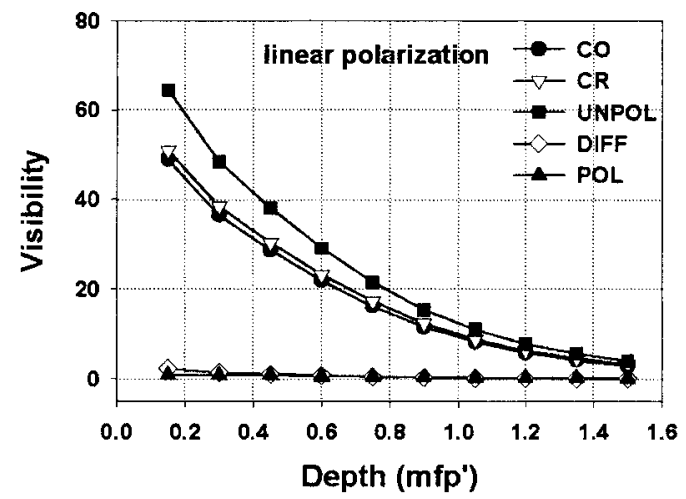

(b)

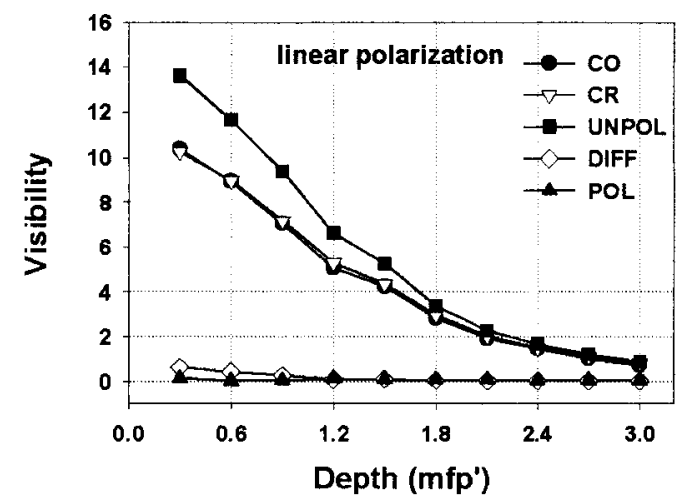

(c)

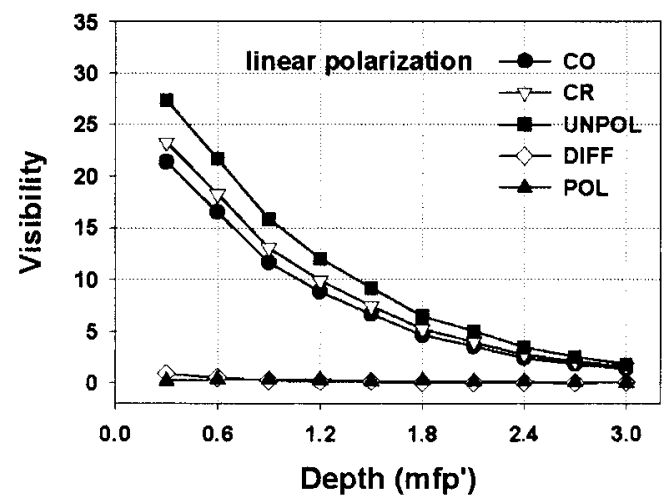

(d)

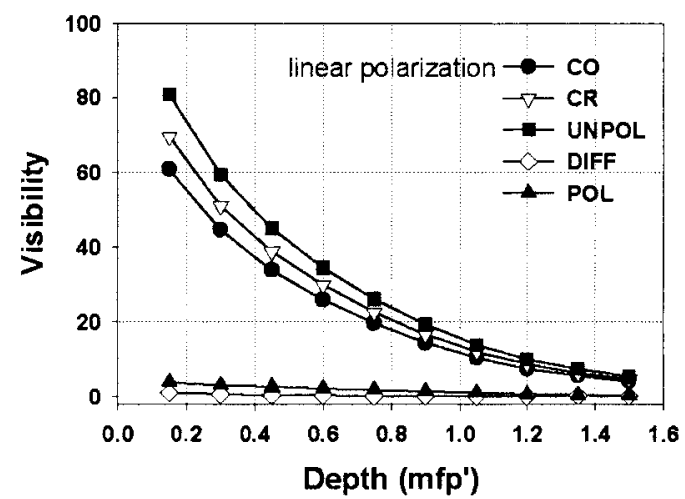

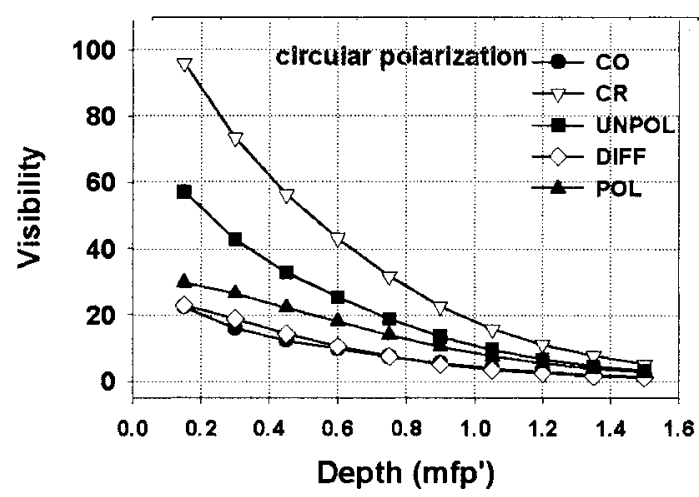
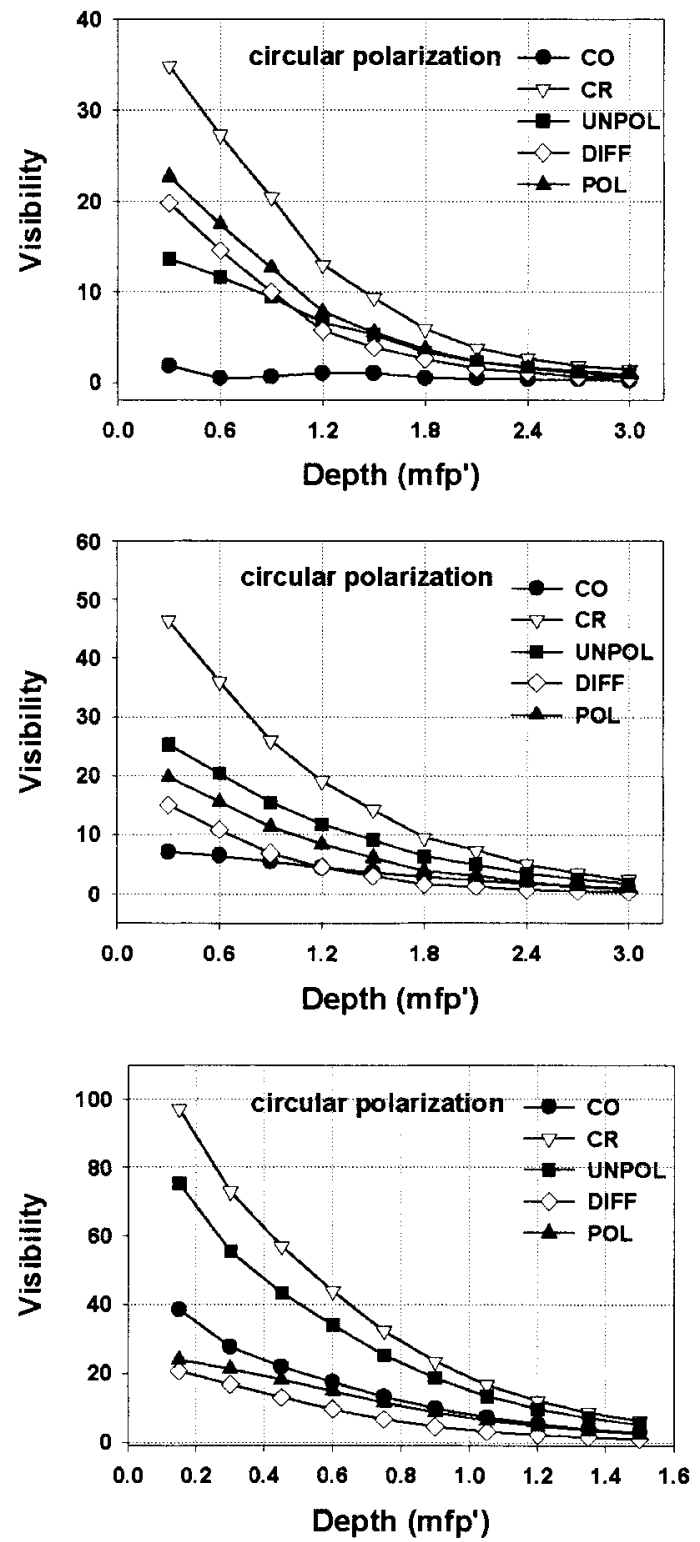

Fig. 4. Effects of background optical properties on polarization image visibility of a scattering target. The optical properties of the turbid media are: (a) $\mu_{s}{ }^{\prime}=3.75 \mathrm{~cm}^{-1}, \mu_{a}=0.1 \mathrm{~cm}^{-1}$, and $g=0.92$; (b) $\mu_{s}{ }^{\prime}=15.0 \mathrm{~cm}^{-1}, \mu_{a}=0.1 \mathrm{~cm}^{-1}$, and $g=0.92 ;$ (c) $\mu_{s}{ }^{\prime}=15.0 \mathrm{~cm}^{-1}, \mu_{a}=$ $0.4 \mathrm{~cm}^{-1}$, and $g=0.92 ;$ (d) $\mu_{s}^{\prime}=3.75 \mathrm{~cm}^{-1}, \mu_{a}=0.1 \mathrm{~cm}^{-1}$, and $g=0.72$.

an increase in background $\mu_{s}{ }^{\prime}$ from 3.75 to $15.0 \mathrm{~cm}^{-1}$ improves the visibility of the absorption target, but an increase in background absorption coefficients from 0.1 to $0.4 \mathrm{~cm}^{-1}$ reduces the visibility of the absorption target.

The reflective target is the most interesting [Fig. 
(a)

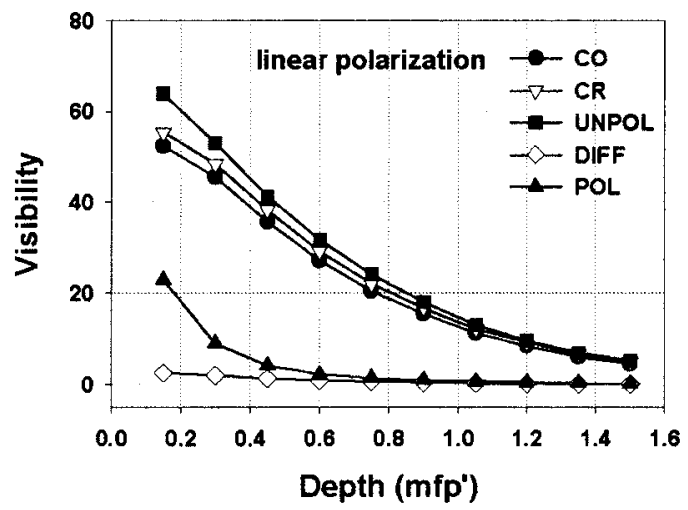

(b)

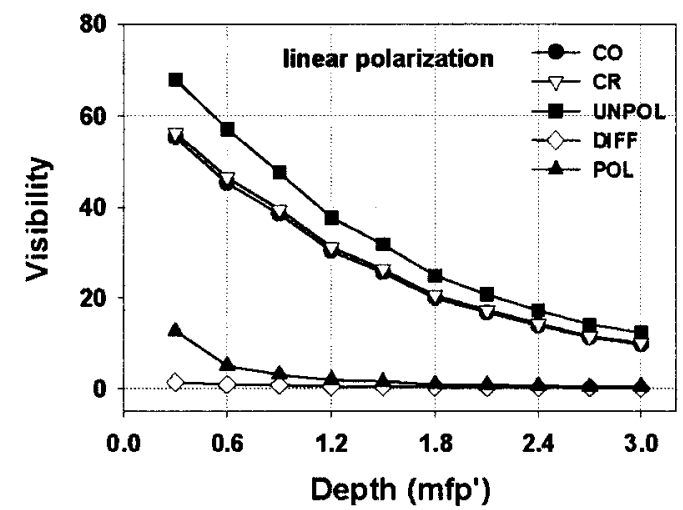

(c)

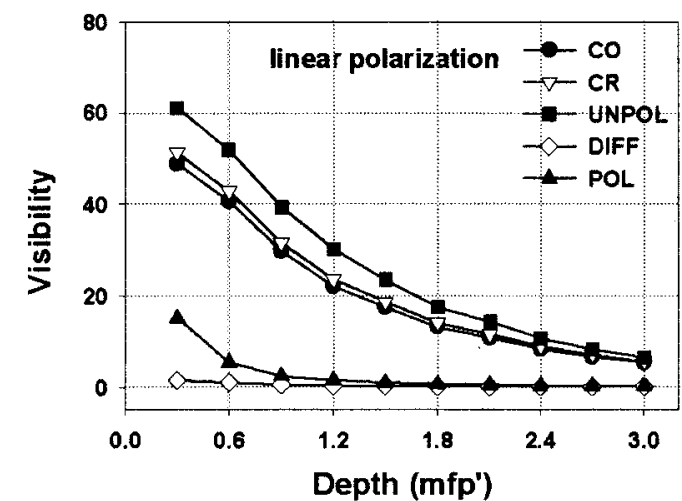

(d)

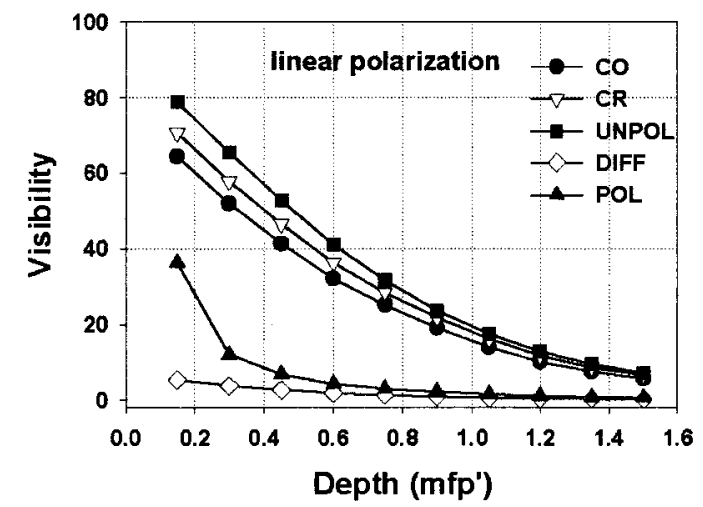

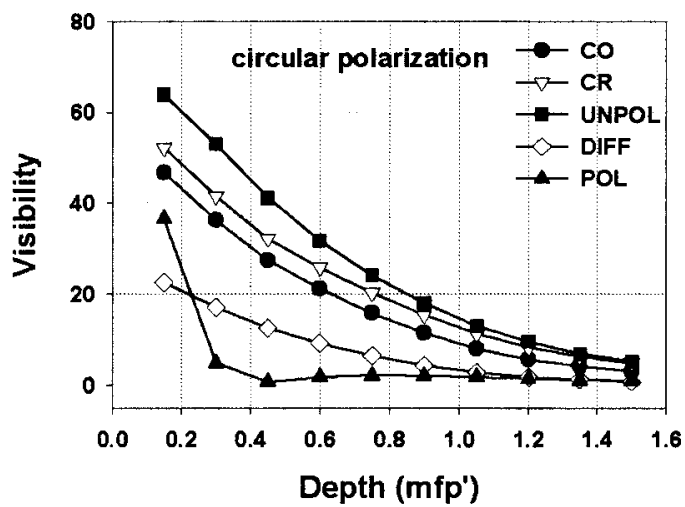
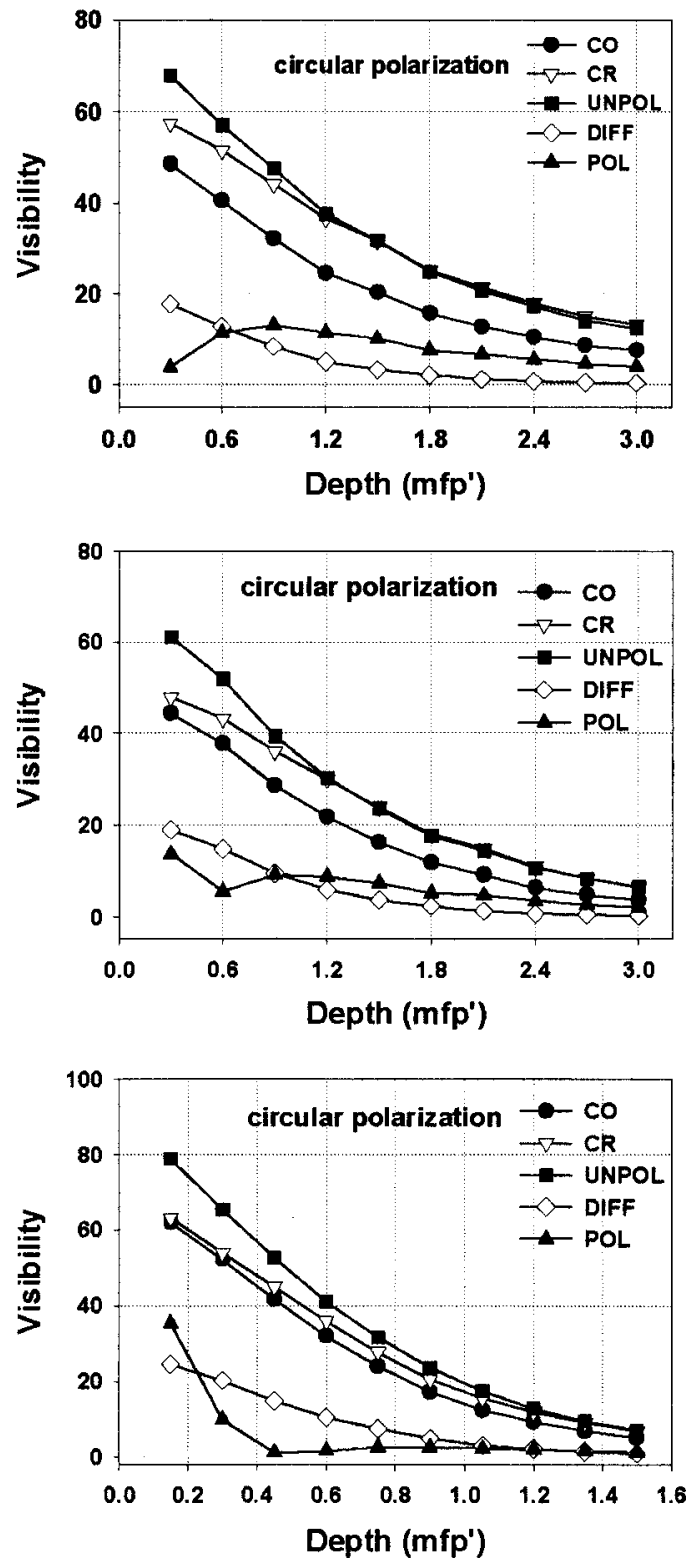

Fig. 5. Effects of background optical properties on the polarization image visibility of an absorption target. The optical properties of the turbid media are the same as in Fig. 4.

3(c)]. At low $\mu_{s}$ and low $\mu_{a}$ the reflection target is brighter than the background, which is similar to a scattering target. However, with an increased $\mu_{s}$ and $\mu_{a}$, the mirror appears dark in the unpolarized image, similar to an absorption target. This is in part due to our choice of $45^{\circ}$ incidence, but it demonstrates the 
(a)

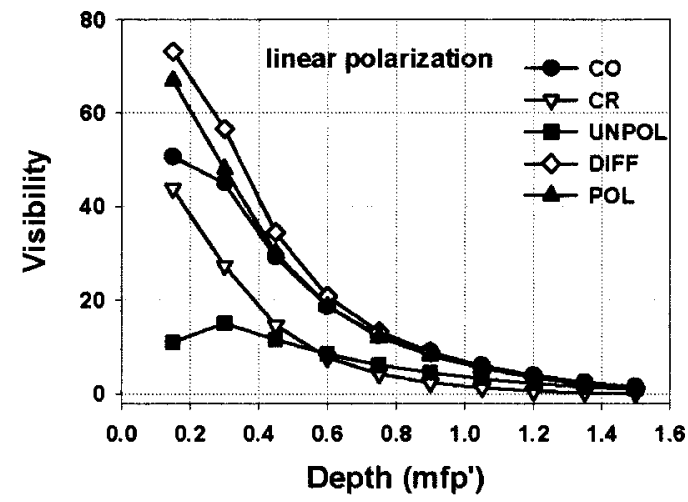

(b)

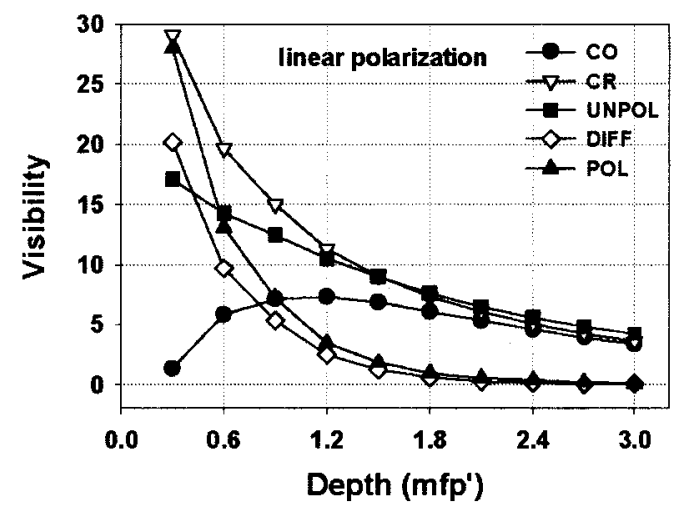

(c)

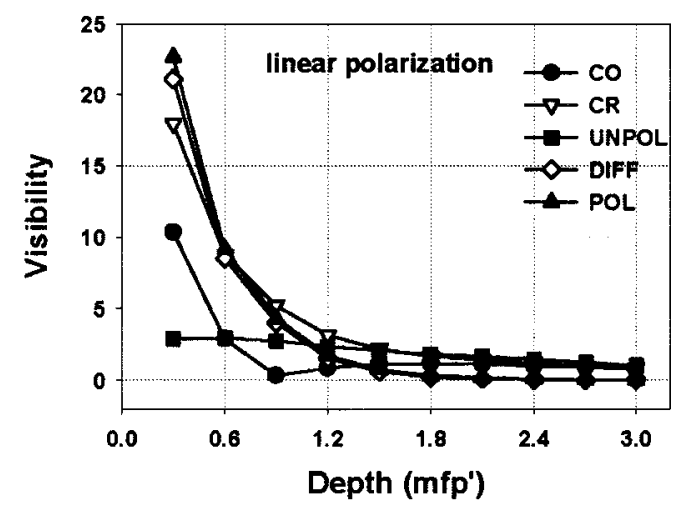

(d)

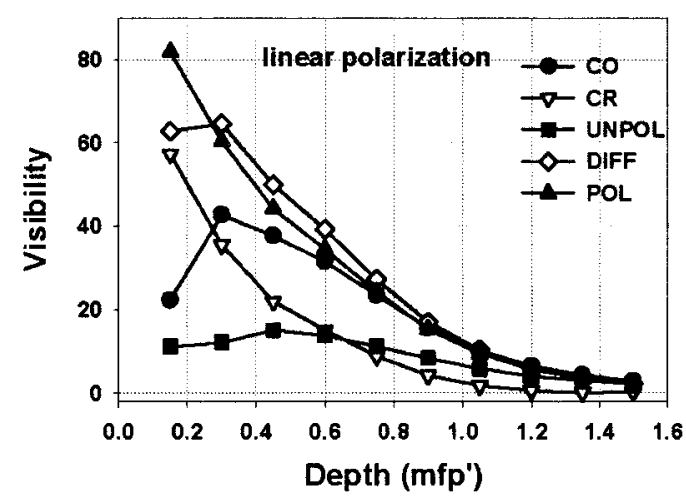

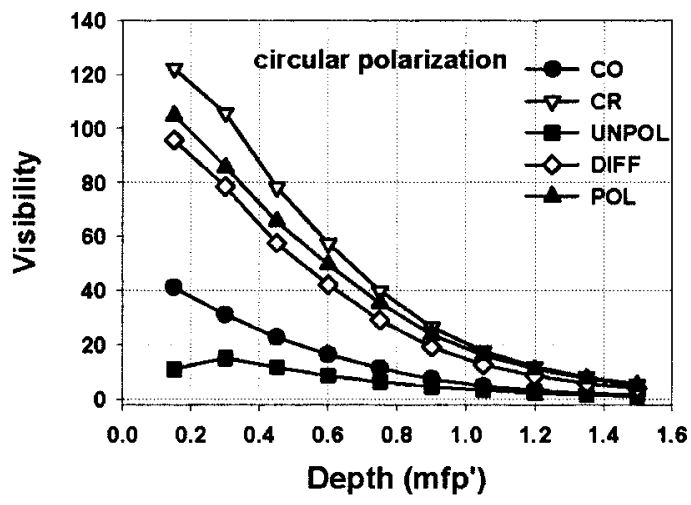
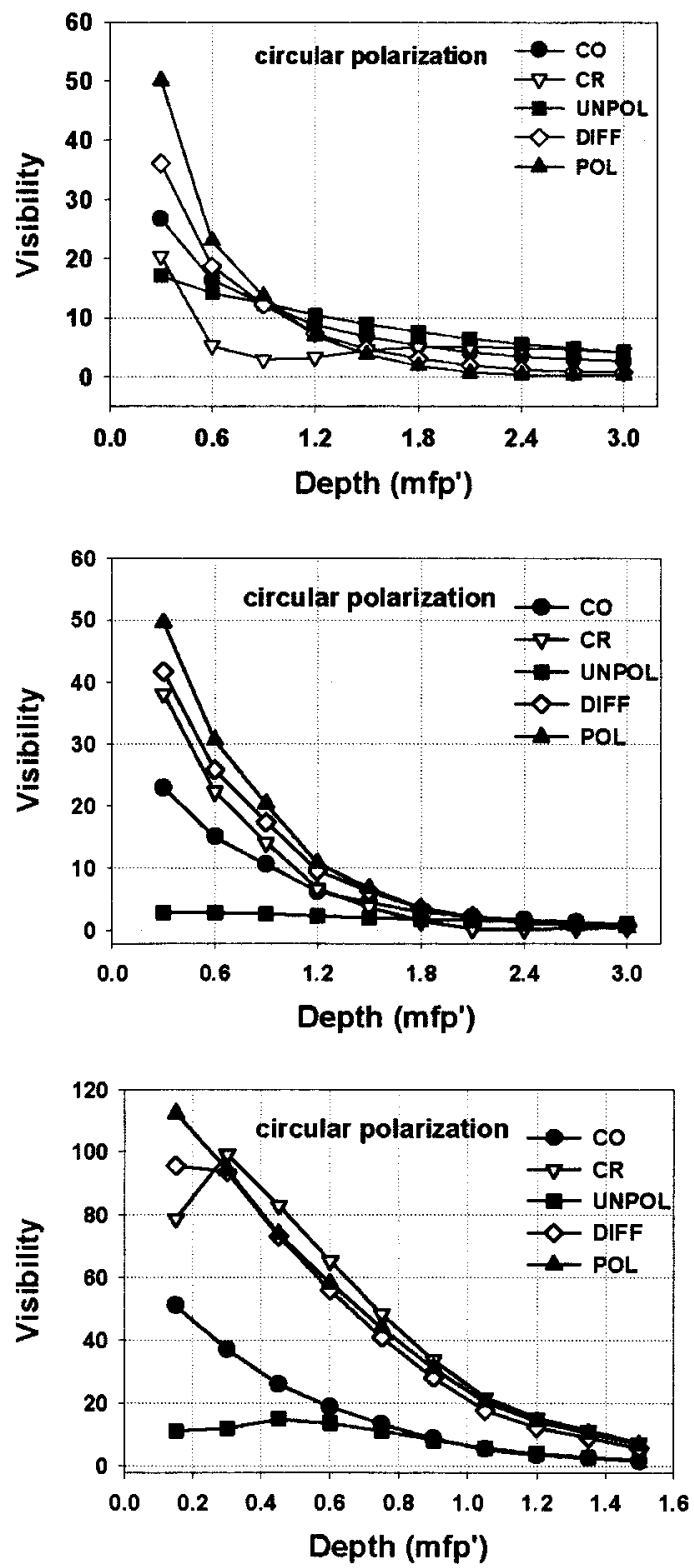

Fig. 6. Effects of background optical properties on the polarization image visibility of a reflection target. The optical properties of the turbid media are the same as in Fig. 4.

potential change in appearance when identical targets are placed in different media. The background optical properties at which the target changes from the bright appearance to the dark appearance depend on the object reflection as well as the illumination angle. 
The anisotropy $g$ has a small effect on unpolarized target visibility. The phantom with a small $g$ consistently outperforms the phantom with a larger $g$. This is probably caused by the difference in scattering coefficients. Achieving the same reduced scattering coefficient $\mu_{s}{ }^{\prime}$ in both phantoms requires a much higher scattering coefficient in the phantom with the larger $g$. As diffusive reflectance from the background is only dependent on $\mu_{s}{ }^{\prime}$, the small $\mu_{s}$ effect is contributed from the target. Because of the limited target depths, the optical signal from the target area is not diffused and cannot be explained with $\mu_{s}{ }^{\prime}$ alone.

\section{B. Effects of Background Optical Properties on Polarized} Images of a Scattering Target

The image visibilities of a scattering target in four different scattering media are shown in Fig. 4. Under linearly polarized illumination, the scattering target achieves the best visibility in the unpolarized image. Changes in background $\mu_{s}{ }^{\prime}, \mu_{a}$, and $g$ have little effect on the relative behavior of polarization images for the scattering targets. The scattering target appears nearly identical in both linear CO and linear CR images because both the target and the background present highly depolarized light. The linear DIFF and POL images produce the worst visibility because the linear CO and CR components are nearly canceled by each other. As the changes in background $\mu_{s}{ }^{\prime}$ and $\mu_{a}$ have little effect on the component linear CO and CR images, they also show little impact on linear DIFF and POL images. A decrease in $g$ value, however, does improve the performance of the linear POL image. The improvement is insignificant with regard to target detection, but should prove insightful when the target or phantom optical properties are unknown.

The CO and CR images are different under circular illumination because the background shows a preference for the $\mathrm{CO}$ due to polarization memory while the target highly depolarizes the light. Because the backscattered intensity is higher from the scattering target, the result is that the target and background values are much closer in the $\mathrm{CO}$ component, whereas they are significantly different in the CR component. Under circumstances in which the background $\mu_{s}{ }^{\prime}$ is high enough, this can make the scattering object nearly invisible in circular CO images [Fig. 4(b)]. Conversely, the circular CR image shows an improvement over unpolarized detection and any other polarization components despite changes in phantom $\mu_{s}{ }^{\prime}$ and $\mu_{a}$. In addition, the performance enhancement by using a circular CR component is greatly affected by sample anisotropy. For phantoms with larger $g$ values, the greater polarization memory effect leads to better visibility improvement in the circular CR image.

The circular POL image is consistently better than DIFF for the scattering target, which indicates that the depolarization information is revealing more about the target than the difference information. Here the increase in $g$ pushes the background copolarization higher while the scattering target still maintains a copolarization near 50\%. Thus a higher $g$ leads to a relative increase in visibility of the scattering target under circular POL, the opposite effect of that seen with linear polarization. As the background scattering increases [Fig. 4(b)], image visibilities of both circular DIFF and POL images can surpass that of the unpolarized image. When the background absorption coefficient increases [Fig. 4(c)], the circular DIFF or POL image falls behind the unpolarized image again. However, a careful examination of Fig. 3 indicates that such changes in relative performance are largely due to the change in the unpolarized image. Despite significant changes in background optical properties $\left(\mu_{s}{ }^{\prime}, \mu_{a}\right.$, and $\left.g\right)$, the absolute and POL and DIFF values are relatively stable for both linearly and circularly polarized light. This result indicates that the DIFF and POL calculations can partially compensate for the changes induced by variations in background optical properties.

\section{Effects of Background Optical Properties on Polarized Images of an Absorption Target}

The image visibilities of an absorption target in four different scattering media are shown in Fig. 5. Similar to the scattering target, all linear polarization images produce worse visibility than the unpolarized image. The absorbing target is almost equally visible in linear CO and CR images owing to the nearly 50\% background copolarization. Therefore the linear DIFF and POL have minimal visibility. The linear CR has slightly better visibility than the linear $\mathrm{CO}$ at small scattering or large absorption. This may be partially attributed to the fact that linearly CO light has a small advantage over linearly CR light from regions above the object. ${ }^{30}$ It is noted that the linear POL has unusually large values at small target depths. An examination of the polarized light intensities from the target area indicates that such an unusual POL value is probably caused by a small residual surface reflection from the absorption target. The background optical properties have the same effects on the polarization images as on the unpolarized image. A larger $\mu_{s}{ }^{\prime}$ leads to better visibility in linear $\mathrm{CO}$ and CR images; whereas a higher $\mu_{a}$ or $g$ leads to lower visibility in these images.

The absorbing target shows little improvement in visibility in circular polarization images because the light signal in both the target and the background regions are the result of the background scattering process. The circular $\mathrm{CR}$ visibility approaches that achieved in the unpolarized image at large $\mu_{s}{ }^{\prime}$ and high anisotropy g. Circular POL values are relatively stable. Circular DIFF visibility is affected by background $\mu_{s}{ }^{\prime}$. At $\mu_{s}{ }^{\prime}=3.75 \mathrm{~cm}^{-1}$, circular DIFF is better than circular POL; whereas at $\mu_{s}{ }^{\prime}=15.0 \mathrm{~cm}^{-1}$, it is worse than circular POL. The background absorption has a similar effect on all images other than POL. It is worth noting that the absorbing target appears bright in the circular POL image, revealing that light in the target region has a higher copolarization than the background. 
D. Effects of Background Optical Properties on Polarized Images of a Reflective Target

Figure 6 shows the image visibilities of a reflective target in four different scattering media. Compared with scattering and absorption targets, the reflection target displays sophisticated behavior in polarization images. In linear polarization images, $\mathrm{CO}$ is superior to $\mathrm{CR}$, and the DIFF offers the best overall visibility in the phantom with low, $3.75 \mathrm{~cm}^{-1}, \mu_{s}^{\prime}$ [Fig. 6(a)]. Increasing $\mu_{s}{ }^{\prime}$ to $15 \mathrm{~cm}^{-1}$ dramatically reverses the behavior. Linear CR and unpolarized images then offer the best visibility. Reducing the absorption coefficient greatly reduces the visibility of linear $\mathrm{CR}$ and the unpolarized image. However, the linear DIFF and POL are less affected by the $\mu_{a}$ change. The change of scattering anisotropy $g$ [Fig. 6(d)] has little effect on the performance of the linear polarization images except at small depths due to surface effects.

In circular polarization images, the circular DIFF and POL are much better than their linear counterparts. They provide significant visibility enhancement over the unpolarized images at small $\mu_{s}{ }^{\prime}$ of $3.75 \mathrm{~cm}^{-1}$. At larger $\mu_{s}{ }^{\prime}$, they still offer better visibility until $\sim 1 \mathrm{mfp}^{\prime}$ depth where the unpolarized image starts to outperform all polarization components. Similar to linear polarization, the circular DIFF and POL are insensitive to changes in $\mu_{a}$ and become the best polarization component because of a decrease in unpolarized visibility. Also similar to linear polarization, the background $\mu_{s}{ }^{\prime}$ and $\mu_{a}$ have a dramatic impact on circular $\mathrm{CO}$ and CR components. At $\mu_{s}^{\prime}=$ $3.75 \mathrm{~cm}^{-1}$, circular CR gives the best image visibility among all the images; while it becomes the worse component up to $1.5 \mathrm{mfp}^{\prime}$ at higher $\mu_{s}{ }^{\prime}$ of $15.0 \mathrm{~cm}^{-1}$. It is significantly recovered when the background $\mu_{a}$ increases to $0.4 \mathrm{~cm}^{-1}$ from $0.1 \mathrm{~cm}^{-1}$. The polarization memory has a greater impact on circular polarization images than on linear polarization images. A high $g$ value leads to enhanced visibility in circular $\mathrm{CR}$ and reduced visibility in circular CO. Because the mirror appears bright at low $\mu_{s}{ }^{\prime}$ and dark at high $\mu_{s}{ }^{\prime}$, the best linear and circular components reverse. This is a strong indication that reflection targets should appear differently depending on the properties of the media and the target reflectance. As with the scattering target, the DIFF and POL are quite stable despite the behavior of the components.

\section{Conclusion}

Several important issues have been clarified in this study. Within the context of imaging, the target visibility depends on the dynamic interactions between the light intensity captured from the background and from the object. In polarization imaging, it is important to remember that the polarization behavior of both the background behavior and the target determine the imaging results. We found that different types of target appear differently in the polarization image. ${ }^{25,26}$ More importantly, target visibility is greatly affected by the optical scattering and absorption properties of the background medium, and such effects depend on the target type. These results have several implications. First, the background optical properties and object types should be considered when applying polarization imaging to enhance image visibility. As an example, Fig. 6 shows that circularly cross-polarized light provides superior image enhancement for reflection targets at small background scattering [Fig. 6(a)], but becomes inferior at large background scattering [Fig. 6(c)]. Second, polarization imaging can be used to identify different types of objects based on their different behaviors as indicated in Fig. 2. Finally, the relative image visibility among different polarization components may reveal certain background optical properties if the target type is known. Using the reflection target as an example, if the circular $\mathrm{CO}$ has a better performance than the circular CR, it may indicate that the background medium is high scattering and low absorption. However, it is worth mentioning that pure target types are used in this study. A real target is probably a combination of optical scattering, absorption, and reflection although it may be dominant in one of these aspects. Further studies are necessary to investigate the capability and limitation of polarization imaging techniques for such target identification.

The authors acknowledge financial support by a research board grant from the University of Missouri-Columbia.

\section{References}

1. G. P. Konnen, Polarized Light in Nature (Cambridge U. Press, 1985).

2. T. W. Cronin, N. Shashar, R. L. Caldwell, J. Marshall, A. G. Cheroske, and T. H. Chiou, "Polarization vision and its role in biological signaling," Integr. Comp. Biol. 43, 549-548 (2003).

3. S. G. Demos and R. R. Alfano, "Optical polarization imaging," Appl. Opt. 36, 150-155 (1997).

4. G. D. Lewis, D. L. Jordan, and P. J. Roberts, "Backscattering target detection in a turbid medium by polarization discrimination," Appl. Opt. 38, 3937-3944 (1999).

5. S. G. Demos, H. B. Radousky, and R. R. Alfano, "Deep subsurface imaging in tissues using spectral and polarization filtering," Opt. Express 7, 23-28 (2000).

6. S. L. Jacques, J. R. Roman, K. Lee, "Imaging superficial tissues with polarized light," Lasers Surg. Med. 26, 119-129 (2000).

7. S. Morgan and I. Stockford, "Surface-reflection elimination in polarization imaging of superficial tissue," Opt. Lett. 28, 114116 (2003).

8. V. Backman, R. Gurjar, K. Badizadegan, I. Itzkan, R. R. Dasari, L. T. Perelman, and M. S. Feld, "Polarized light scattering spectroscopy for quantitative measurement of epithelial cellular structures in situ," IEEE J. Sel. Top. Quantum Electron. 5, 1019-1026 (1999).

9. M. P. Rowe and E. N. Pugh, Jr., J. S. Tyo, and N. Engheta, "Polarization-difference imaging: a biologically inspired technique for observation through scattering media," Opt. Lett. 20, 608-610 (1995).

10. J. S. Tyo, M. P. Rowe, E. N. Pugh, Jr., and N. Engheta, "Target detection in optically scattered media by polarizationdifference imaging," Appl. Opt. 35, 1855-1870 (1996).

11. Y. Y. Schechner, S. G. Narasimhan, and S. K. Nayar, "Polarization-based vision through haze," Appl. Opt. 42, 511525 (2003). 
12. L. B. Wolff, "Polarization-based material classification from specular reflection," IEEE Trans. Pattern Anal. Mach. Intell. 12, 1059-1071 (1990).

13. L. B. Wolff and T. E. Boult, "Constraining object features using a polarization reflectance model," IEEE Trans. Pattern Anal. Mach. Intell. 13, 635-657 (1991).

14. H. Chen and L. B. Wolff, "Polarization phase-based method for material classification in computer vision," Int. J. Comput. Vis. 28, 73-83 (1998).

15. S. G. Demos, A. J. Papadopoulos, H. Savage, A. S. Heerdt, S. Schantz, and P. R. Alfano, "Polarization filter for biomedical tissue optical imaging," Photochem. Photobiol. 66, 821-825 (1997).

16. S. L. Jacques, J. R. Roman, and K. Lee, "Imaging superficial tissues with polarized light," Lasers Surg. Med. 26, 119-129 (2000).

17. S. L. Jacques, J. C. Ramella-Roman, and K. Lee, "Imaging skin pathology with polarized light," J. Biomed. Opt. 7, 329-340 (2002).

18. G. D. Gilbert and J. C. Pernicka, "Improvement of underwater visibility by reduction of backscatter with a circular polarization technique," Appl. Opt. 6, 741-746 (1967).

19. G. D. Gilbert and J. C. Pemicka, "Improvement of underwater visibility by reduction of backscatter with a circular polarization technique," Appl. Opt. 6, 741-746 (1967).

20. V. Sankaran, J. T. Walsh, and D. J. Maitland, "Comparative study of polarized light propagation in biologic tissues," J. Biomed. Opt. 7, 300-306 (2002).

21. F. C. MacKintosh, J. X. Zhu, D. J. Pine, and D. A. Weitz,
"Polarization memory of multiply scattered light," Phys. Rev. B 40, 9342-9345 (1989).

22. A. D. Kim and M. Moscoso, "Backscattering of circularly polarized pulses," Opt. Lett. 27, 1589-1991 (2002).

23. X. Ni and R. R. Alfano, "Time-resolved backscattering of circularly and linearly polarized light in a turbid medium," Opt. Lett. 29, 2773-2775 (2004).

24. S. A. Kartazayeva, X. Ni, and R. R. Alfano, "Backscattering target detection in a turbid medium by use of circularly and linearly polarized light," Opt. Lett. 30, 1168-1170 (2005).

25. G. Yao, "Differential optical polarization imaging in turbid media with different embedded objects," Opt. Commun. 241, 255-261 (2004).

26. R. Nothdurft and G. Yao, "Expression of target optical properties in subsurface polarization-gated imaging," Opt. Express 13, 4185-4195 (2005).

27. N. Ghosh, H. S. Patel, and P. K. Gupta, "Depolarization of light in tissue phantoms-effect of a distribution in the size of scatterers," Opt. Express 11, 2198-2205 (2003).

28. R. Nothdurft and G. Yao, "Reveal obscured subsurface object using laser speckle imaging," Opt. Express 13, 10034-10039 (2005).

29. F. Fabbri, M. A. Franceschini, and S. Fantini, "Characterization of spatial and temporal variations in the optical properties of tissue like media with diffuse reflectance imaging," Appl. Opt. 42, 3063-3072 (2003).

30. S. P. Morgan and M. E. Ridgway, "Polarization properties of light backscattered from a two layer scattering medium," Opt. Express 7, 395-402 (2000). 